\title{
Patient-specific stress analyses in the ascending thoracic aorta using a finite-element implementation of the constrained-mixture theory
}

\author{
S. Jamaleddin Mousavi · Stéphane Avril
}

the date of receipt and acceptance should be inserted later

\begin{abstract}
It is now a rather common approach to perform patient-specific stress analyses of arterial walls using finite-element models reconstructed from gated medical images. However this requires to compute for every Gauss point the deformation gradient between the current configuration and a stressfree reference configuration. It is technically difficult to define such a reference configuration and there is actually no guarantee that a stressfree configuration is physically attainable due to the presence of internal stresses in unloaded soft tissues. An alternative framework was proposed by Bellini et al., 2014. It consists of computing the deformation gradients between the current configuration and a prestressed reference configuration. We present here the first finite-element results based on this concept using the Abaqus software. The reference configuration is set arbitrarily to the in vivo average geometry of the artery, which is obtained from gated medical images and is assumed to be mechanobiologically homeostatic. For every Gauss point, the stress is split additively into the contributions of each individual load-bearing constituent of the tissue, namely elastin, collagen, smooth muscle cells. Each constituent is assigned an independent prestretch in the reference configuration, named the deposition stretch. The outstanding advantage of the present approach is that it simultaneously computes the in situ stresses existing in the reference configuration and predicts the residual stresses that occur after
\end{abstract}

S.J. Mousavi ${ }^{1} \cdot$ S. Avril ${ }^{2}$

Mines Saint-Étienne, CIS-EMSE, F-42023 Saint-Étienne, France

INSERM， U1059， SAINBIOSE， F-42023 Saint-Étienne, France

Université de Lyon, F-69000 Lyon, France

${ }^{1}$ Corresponding author E-mail: Jamal.mousavi@emse.fr

${ }^{2}$ E-mail: Avril@emse.fr removing the different loadings applied onto the artery (pressure and axial load). As a proof of concept, we applied it on an ideal thick-wall cylinder and showed that the obtained results were consistent with corresponding experimental and analytical results of wellknown literature. In addition, we developed a patientspecific model of a human ascending thoracic aneurysmal aorta and demonstrated the utility in predicting the wall stress distribution in vivo under the effects of physiological pressure. Finally we simulated the whole process preceding traditional in vitro uniaxial tensile testing of arteries, including excision from the body, radial cutting, flattening and subsequent tensile loading, showing how this process may impact the final mechanical properties derived from these in vitro tests.

Keywords finite-elements - constrained mixture theory · anisotropic behaviour · zero-pressure configuration $\cdot$ residual stresses

\section{Introduction}

For the past few years, scientists have employed different methodologies, such as the theory of continuum mechanics, to investigate the biomechanical behavior of arteries. Important studies demonstrated that mechanical factors may play an essential role in the development of cardiovascular diseases, such as atherosclerosis or aneurysms, which are prevalent causes of many deaths in the western world $[26,28,58]$.

An important aspect of the biomechanical behavior of arteries is the permanent state of biaxial tensile stresses that exists in vivo. The existence of these in situ stresses is well evidenced when arteries are excised during a surgical intervention for instance. Due to the removal of in vivo loadings, the artery experiences an elastic recoiling 
$[10,29]$. Even when these loadings are removed, internal or residual stresses still exist in the tissues. An effect of these internal stresses can be observed in arteries with the well-known open angle experiment [2,18,29]. Residual stresses can be observed in most of soft tissues [36, $60]$, including myocardium $[22,40,57]$ and mitral valve leaflets [44]. The intrinsic reason of this specific mechanical behavior of soft tissues is their composite nature combined with different kinetics of growth and remodeling for each micro-constituent. For instance, in large arteries, while the extracellular matrix (ECM) of the media mainly consists of a complex three-dimensional (3D) network of elastin and collagen fibrils surrounding the smooth muscle cells (SMCs), the ECM in the outer layer (adventitia) is mostly made of thick bundles of collagen.

It is now a rather common approach to perform patientspecific stress analyses in arterial walls using finiteelement (FE) models reconstructed from gated medical images $[20,45,46,52,53]$. There is also nowadays an important endeavor to understand and model computationally the mechanobiological behavior of arteries [37], especially how the fibrobasts and SMCs sense the in situ mechanical stimuli and maintain stresses or stiffness properties at homeostasis $[6,7,8,39]$. However this requires for every Gauss point to compute the deformation gradient between the current configuration and a stressfree reference configuration. It is technically difficult to derive such reference configuration. Moreover, there is actually no guarantee that a stressfree configuration is physically attainable due to the presence of internal stresses after removing the external loads and even after introducing a radial cut.

For specific applications, arterial walls were modeled as a homogenized single layer [33,49]. The in situ stresses existing in vivo are taken into account by determining a stress-free zero-pressure reference configuration. For instance, Riveros et al. [45] proposed an iterative FE model to predict the zero-pressure geometry of patient specific abdominal aortic aneurysms (AAA). The aortic wall was considered as an anisotropic hyperelastic material and the intraluminal thrombus was also considered. The residual stresses were neglected.

One can also find a number of computational models accounting for the bi-layered structure of arteries in the literature $[14,25,35,41,42,50,55,56]$. Maltzahn et al. $[55,56]$ used a finite deformation framework and reported discontinuous axial and circumferential stresses at the interface of the media and adventitia, the maximum circumferential stresses occurring in the media. They obtained non uniform stress distributions across the thickness of the artery but they neglected residual stresses.
Other authors included the residual stresses in their model by defining the reference configuration as the geometry of the artery resulting from the open angle experiment [25]. Doing so and using bi-layer models, Taber et al. [50] and Rachev [42] obtained a roughly uniform distribution of circumferential stress in each arterial layer.

Another type of approach permitting to address this problem is based on the Constrained Mixture Theory (CMT) [30]. Experiments reveal that the net residual stresses in an excised artery likely depend on different residual stresses within individual constituents. This is consistent with the fact that each constituent is deposited at a different time period and with different value, which leads each constituent to have a different deposition stretch. According to the principle of the constrained mixture theory (CMT), all constituents in the mixture are assumed to deform together in the stressed configuration whereas they have their own evolving mass fractions. Indeed, this suggestion is consistent with the idea that elastin is deposited and cross-linked during the perinatal period and its half-life is normally on the order of decades, thus causing it to undergo extensive elastic deformations during normal biological growth $[5,11]$ that will be partially released in the mature artery upon excision. On the other hand, collagen turns over continuously and is likely deposited in the current state of the artery at maturity [29,51]. The extensibility of collagen fibers being rather limited, of the order of $10 \%$ strain, the prestretch of collagen is rather modest compared to the one of elastin. Following the seminal paper of Humphrey and Rajagopal [31], mathematical models and computational frameworks based on the CMT were recently published in several papers, including Bellini et al. [2], Cardamone et al. [5] and Valentín et al. [54]. It was shown that they can successfully predict the zero-pressure configuration and residual stresses. The approach was used successfully for thick cylinders and a FE implementation would permit applying it on patient-specific geometries of arterial walls reconstructed from medical images.

In the present work, the objective is to develop and apply the first FE model based on the CMT concept using the Abaqus software. We applied it first on an ideal thick-wall cylinder and showed that the obtained results were consistent with corresponding experimental and analytical results of well-known literature. In addition, we developed a patient-specific model of a human ascending thoracic aneurysmal aorta (ATAA) and demonstrated the utility in predicting the wall stress distribution in vivo under the effects of physiological pressure. Finally we simulated the whole process preceding traditional in vitro mechanical testing of arter- 
ies, including excision from the body, radial cutting, flattening and subsequent tensile loading, showing how this process may impact the final mechanical properties derived from these in vitro tests.

\section{Material and methods}

\subsection{Constitutive model}

It has been recently demonstrated that $2 \mathrm{D}$ mathematical [2] and 3D computational [54] models based on the CMT are able to successfully predict the behavior of thick-wall arteries concerning zero-pressure configuration, residual stresses and growth and remodeling. In these manuscripts the approach was used successfully to predict the behavior of the thick cylinders. However those studies lacked a 3D demonstration and application on actual ATAA model. Therefore, we propose to develop FE models of arteries using 3D hexahedral or wedge finite elements. At the Gauss points of each element, the passive behavior is assumed hyperelastic and a strain-energy function ( $\mathrm{SEF}$ ) is assumed with decoupled contributions of the purely volumetric contributions and the purely isochoric contributions $[2,25,28$, 54]. The same SEF is assumed for every element across the geometry of the artery, but different material properties can be applied at each layer.

It is assumed that the isochoric part of the SEF can be written as an additive split of different contributions: the contribution of elastin, the passive contribution of SMC if present, and the contribution of collagen fibers (Fig. 1). For the latter, a number of families can be considered. Eventually, the SEF may be written as $[2$, 28]:

$$
\begin{gathered}
W=\rho^{\mathrm{e}} \bar{W}^{\mathrm{e}}\left(\bar{I}_{1}^{\mathrm{e}}\right)+\sum_{i=1}^{n} \rho^{\mathrm{c}_{i}} \bar{W}^{\mathrm{c}_{i}}\left(\bar{I}_{4}^{\mathrm{c}_{i}}\right)+ \\
\rho^{\mathrm{m}} \bar{W}^{\mathrm{m}}\left(\bar{I}_{4}^{\mathrm{m}}\right)+U(J)
\end{gathered}
$$

where superscripts $\mathrm{e}, \mathrm{c}_{i}$ and $\mathrm{m}$ represent respectively the elastin fiber constituent, the constituent made of each of the $n$ possible collagen fiber families and the SMC constituent. In Eq. 1, $\rho^{j}$ refers to mass fraction, $\bar{W}^{j}$ and $U(J)$ stand for the isochoric and volumetric stored elastic energy, depending on the first $\left(\bar{I}_{1}^{j}\right)$ and fourth $\left(\bar{I}_{4}^{j}\right)$ invariants of the related constituents of the mixture $\left(j \in\left\{\mathrm{e}, \mathrm{c}_{i}, \mathrm{~m}\right\}\right)$. Let the mechanical behavior of the elastin constituent be described by a Neo-Hookean strain energy function as in $[5,15,25]$

$\bar{W}^{\mathrm{e}}\left(\bar{I}_{1}^{\mathrm{e}}\right)=\frac{\mu^{\mathrm{e}}}{2}\left(\bar{I}_{1}^{\mathrm{e}}-3\right)$ where $\mu^{\mathrm{e}}$ is a material parameter and has a stress-like dimension, and:

$\bar{I}_{1}^{\mathrm{e}}=\operatorname{tr}\left(\overline{\mathbf{C}}^{\mathrm{e}}\right)$

where $\overline{\mathbf{C}}^{\mathrm{e}}=\overline{\mathbf{F}}^{\mathrm{e} \mathrm{T}} \overline{\mathbf{F}}^{\mathrm{e}}$ denotes the right Cauchy-Green tensor where $\overline{\mathbf{F}}^{\mathrm{e}}=J^{\mathrm{e}(-1 / 3)} \mathbf{F}^{\mathrm{e}}$ is the isochoric part of the deformation in the elastin constituent, with the volume ratio denoted $J^{\mathrm{e}}=\operatorname{det}\left(\mathbf{F}^{\mathrm{e}}\right)>0$ and $\operatorname{det}\left(\overline{\mathbf{F}}^{\mathrm{e}}\right)=1$. Noting that $\mathbf{F}^{\mathrm{e}}=\mathbf{F G}_{h}^{\mathrm{e}}$ where $\mathbf{F}$ is the corresponding deformation gradient of the mixture and $\mathbf{G}_{h}^{\mathrm{e}}$ is the deposition stretch of elastin with respect to the reference configuration $[2,5]$. Therefore, using the CMT-based model it is assumed that all constituents in the mixture deform together in the stressed configuration while each constituent has a different "total" deformation gradient based on its own deposition stretch.

The SEF of the passive SMCs and collagen contributions is described using an exponential expression such as $[2,5,45,46]$ :

$\bar{W}^{k}\left(\bar{I}_{4}^{k}\right)=\frac{D_{1}^{k}}{2 D_{2}^{k}}\left[\exp \left(D_{2}^{k}\left(\bar{I}_{4}^{k}-1\right)^{2}\right)-1\right]$

where $k \in\left\{\mathrm{c}_{i}, \mathrm{~m}\right\} . D_{1}^{\mathrm{k}}$ and $D_{2}^{\mathrm{k}}$ are stress-like and dimensionless material parameters, respectively, and can take different values when fibers are under compression or tension [4]. $\bar{I}_{4}^{\mathrm{k}}=J^{k^{(-2 / 3)}} I_{4}^{\mathrm{k}}$ which is greater than one when fiber is under tension and less than one when fiber is under compression. Noting that $J^{\mathrm{k}}=\operatorname{det}\left(\mathbf{F}^{\mathrm{k}}\right)$ and $I_{4}^{\mathrm{k}}$ is the fourth invariant which can be written such as:

$I_{4}^{k}=G_{h}^{k^{2}} \mathbf{C}: \mathbf{M}^{k} \otimes \mathbf{M}^{k}$

where $G_{h}^{k}, k \in\left\{\mathrm{c}_{i}, \mathrm{~m}\right\}$, is the specific deposition stretch of each collagen fiber family or SMCs, with respect to the reference configuration. $\mathbf{M}^{k}, k \in\left\{\mathrm{c}_{i}, \mathrm{~m}\right\}$, denotes a unit vector along the dominant orientation of anisotropy in the reference configuration of the constituent made of the $i$ th family of collagen fibers or of SMCs. For SMCs, $\mathbf{M}^{m}$ coincides with the circumferential direction of the vessel in the reference configuration while for the $i$ th family of collagen fibers $\mathbf{M}^{c_{i}}=\left[\begin{array}{ll}0 \sin \alpha^{i} \cos \alpha^{i}\end{array}\right]$, where $\alpha^{i}$ is the angle of the $i$ th family of collagen fibers with respect to the axial direction. $\mathbf{C}=\mathbf{F}^{\mathrm{T}} \mathbf{F}$ is the right Cauchy-Green stretch tensor of the arterial wall mixture $[2,5]$.

The volumetric component of the SEF is written such as [45]:

$U(J)=\kappa(J-1)^{2}$

where $\kappa$ is the bulk modulus and $J=\operatorname{det}(\mathbf{F})$ is the Jacobian. 
The second Piola-Kirchhoff stress tensor can be obtained as:

$$
\begin{aligned}
\mathbf{S}= & 2 \rho^{\mathrm{e}} \frac{\partial \bar{W}^{\mathrm{e}}}{\partial \bar{I}_{1}^{\mathrm{e}}} \frac{\partial \bar{I}_{1}^{\mathrm{e}}}{\partial \mathbf{C}}+\sum_{i=1}^{n} 2 \rho^{\mathrm{c}_{i}} \frac{\partial \bar{W}^{\mathrm{c}_{i}}}{\partial \bar{I}_{4}^{\mathrm{c}_{i}}} \frac{\partial \bar{I}_{4}^{\mathrm{c}_{i}}}{\partial \mathbf{C}}+ \\
& 2 \rho^{\mathrm{m}} \frac{\partial \bar{W}^{\mathrm{m}}}{\partial \bar{I}_{4}^{\mathrm{m}}} \frac{\partial \bar{I}_{4}^{\mathrm{m}}}{\partial \mathbf{C}}+J p \mathbf{C}^{-1}
\end{aligned}
$$

where $p=\mathrm{d} U / \mathrm{d} J$ is the hydrostatic pressure. The Cauchy stress tensor is derived from the second Piola-Kirchoff stress as $[24,25]$

$\boldsymbol{\sigma}=J^{-1} \mathbf{F S F}{ }^{\mathrm{T}}$

\subsection{Finite-Element implementation}

The proposed model is implemented within the commercial FE software Abaqus [23] through a coupled user material subroutine (UMAT) [16,38]. A 3D structural mesh made of hexahedral elements with eight nodes and eight Gauss points is reconstructed across the wall of the artery. The mesh is structural, which means that the edges of each hexahedron are locally aligned with the material directions of the artery: radial, circumferential and axial. For non-perfectly cylindrical geometries, the radial direction is defined as the outward normal direction to the luminal surface, the axial direction is defined as the direction parallel to the luminal centerline in the direction of the blood flow, and the circumferential direction is perpendicular to the two previously defined directions. It is assumed that each element is a mixture of elastin, collagen and SMCs with mass fractions varying regionally.

For each simulation the deposition stretch of elastin should be determined and a specific algorithm had to be implemented for this. Indeed, it is well-known that elastin is produced primarily during the perinatal period and it is mainly stable thereafter for at least a few decades $[11,34]$. Thus as the artery enlarges from infancy to adulthood, it undergoes large multiaxial stretches. In the contrary, collagen and SMCs are continuously produced and replaced during lifetime [34]. Therefore, elastin experiences a higher prestretch (including both developmental and deposition stretches) in comparison with collagen fibers and SMCs which are deposited at maturity. To characterize experimentally the deposition stretches of collagen fibers and SMCs, a method was proposed by [3]. First the specimen is stretched to its in vivo axial length and is pressurized correspondingly. After infusing the sample by appropriate amount of porcine pancreatic elastase for some minutes the elastic fibers are digested [17]. Therefore, measured changes in unloaded length and outer diameter following elastase treatment are then combined with homeostatic values of the biaxial stretches obtained from the distensionextension data to provide a range for the deposition stretch of collagen fibers and smooth SMCs [3]. Therefore, according to those experimental data $[2,3]$, deposition stretches of the SMCs constituent, $G_{h}^{\mathrm{m}}$, and of the collagen constituents, $G_{h}^{\mathrm{c}_{i}}$, are assumed $\sim 1.1$. Refined values around 1.1 can be deduced using inverse approach and biaxial test data [4]. The deposition stretches of the elastin constituent are defined iteratively. The axial deposition stretch of elastin is assigned such as being able to induce the usual axial recoil that can be measured when the artery is excised (this axial stretch may depend on species, on the type of arteries, on the age, etc [27]). Assuming a cylindrical geometry and incompressibility, the structure of the prestretch tensor in matrix notation can be written such as

$\mathbf{G}_{h}^{\mathrm{e}}=\left[\begin{array}{ccc}\frac{1}{\lambda_{\theta} \lambda_{z}^{i v}} & 0 & 0 \\ 0 & \lambda_{\theta} & 0 \\ 0 & 0 & \lambda_{z}^{i v}\end{array}\right]$

In the first step, circumferential component of deposition stretch of elastin is set to one, $\lambda_{\theta}=1$. Therefore, by applying diastolic pressure and taking into account deposition stretches of collagen and SMCs mentioned above, an FE analysis is performed for the first step. The deformation gradient of elastin, $\mathbf{F}^{\mathrm{e}}$, is recorded at the end of each step and assigned as the deposition stretch tensor of the elastin component in the next step until it converges to a stable value. The decision that deposition stretch tensor of the elastin is stable is made according to average nodal displacements across the arterial wall, $U$. So at the end of each step (at convergence of the algorithm), $U$ must be less than a prescribed tolerance, $\xi$, which is set to $1 \%$ of the wall thickness in the present work. This entire algorithm to calibrate the deposition stretch tensor of elastin is summarized in a flowchart shown in Fig. 2.

After calibrating the deposition stretch tensor of the elastin component, FE analyses can be performed to deduce the response of the artery to mechanical actions. For instance, if we set the luminal pressure to zero, we can deduce the pressure-free geometry of the artery including the distribution of residual stresses across the wall.

2.3 Application to predict the tension-inflation and opening angle response of murine arterial segments

The FE model is validated against data available for a thick-wall cylinder (outer diameter $=697 \mu \mathrm{m}$ ) subjected to pressures $P_{i}$ varying from 0 to $140 \mathrm{mmHg}$, at 
three different axial stretches $\left(\lambda_{z}=\{1.47,1.55,1.63\}\right)$ [2]. The cylinder wall is meshed with 7500 hexahedral elements, with 10 elements across the thickness. Axial and circumferential displacements of both ends of the cylinder are blocked, only radial displacements are allowed. Mass fractions $\rho^{\mathrm{e}}, \rho^{\mathrm{c}_{i}}$ and $\rho^{\mathrm{m}}$ introduced in the expression of the SEF in Eq. 1 are the ones reported by [2]. Material parameters are reported in Table 1. For every pressure $P_{i}$ and every axial stretch $\lambda_{z}^{\text {iv }}$, the FE analysis with the CMT-based model is used to derive the outer diameter of the artery, denoted $D$, and the reduced axial force, denoted $F$, permitting to plot $P-D$ and $F-P$ curves for the three applied axial stretches. The reduced axial force is derived by subtracting $P_{i} \pi r_{i}^{2}$ from calculated numerical axial forces, where $r_{i}$ is the actual inner radius of the cylinder [25]. Finally a FE analysis is performed on a small ring of the same arterial model, this time with no pressure, no axial stretch and with a radial cut, permitting to simulate the open angle experiment and to estimate the opening angle resulting from the release of internal stresses. In this case, boundary conditions are assigned in one of the cut surfaces as shown in Fig. 3

\subsection{Application to predict the patient-specific} distribution of stresses in human ATAAs

To demonstrate its applicability to predict patient-specific wall stresses, the model was employed onto a human ascending thoracic aortic aneurysm (ATAA). An ATAA specimen and the preoperative CT scan of the patient were obtained after informed consent from a donor undergoing elective surgery for ATAA repair at CHU-SE (Saint-Etienne, France). The lumen of the aneurysm was clearly visible in the DICOM file, but detection of the aneurysm surface was not possible automatically. A non-automatic segmentation of the CT image slices was performed using MIMICS (v. 10.01, Materialise NV) to reconstruct the ATAA geometry. The reconstructed geometry was meshed with 53820 hexahedral elements. A wall thickness of $2.38 \mathrm{~mm}$ was defined evenly in the reference configuration, yielding an average thickness of $2.67 \mathrm{~mm}$ at zero pressure, which corresponded to the measured thickness on the supplied sample. The thickness was indeed measured homogeneous on the excised aneurysm of this patient in vitro using a digital caliper. As explained in $[52,53]$ the piece of artery was put between two plastic plates and the thickness of the artery and the plates was measured. Then subtracting the thickness of the two plates from the obtained value delivered artery thickness. The mesh comprised five elements across the thickness. Material parameters (reported in Table 2) such as deposition stretch of collagen and exponents were taken from literature $[2,5]$ and others were calibrated with data of our group [12]. The reference configuration was defined with a luminal pressure of $80 \mathrm{mmHg}$ which corresponded to the diastolic pressure. An axial prestretch (deposition stretch) of $\lambda_{z}^{\text {iv }}=1.3$ was defined for the elastin component and the deposition stretches of collagen and SMC components were set to 1.1. The circumferential deposition stretch of elastin was determined using the iterative algorithm shown in Fig. 2. After setting the determined deposition stretch to all the elements, the zero-pressure geometry was then derived by performing an FE analysis with the model with a luminal pressure set to zero (free edge) as boundary conditions. The distribution of wall stresses at systole was also derived by setting the luminal pressure to $120 \mathrm{mmHg}(16 \mathrm{kPa})$ in the model. In each case, the same boundary conditions were assigned to both ends of the ATAA model, allowing only radial displacements and blocking displacements in the other two directions (circumferential and axial).

The aortic arch motion is linked to that of the heart left ventricle through the aortic root. This motion generates axial stresses in the ascending aorta which may induce in turn higher stress levels in the aortic arch. In this patient-specific analysis this complex boundary conditions of pulling or twisting are first ignored because these data were not available for this patient in vivo. Afterwards, in order to investigate the effects of ventricular contraction on the stress distribution, in vivo measurements of pulling and twist angle of Beller et al. $[1,19]$ are adopted. An axial displacement of 8.9 $\mathrm{mm}$ and a rotation angle of $6^{\circ}$ are applied onto the end of the ATAA model [19].

\subsection{Application to predict the response of human ATAA strips to uniaxial tensile tests}

A third application of the model is to simulate uniaxial tensile tests on strips of arteries considering the mechanical effects of excising and cutting these strips from the in vivo tissue. The reference configuration being set arbitrarily as the one of the artery in the in vivo conditions of diastole, before modeling the tensile test on a strip, we have to model first the elastic recoiling occurring after taking the strip from the artery which is initially pressurized and axially stretched, and second the flattening of the tissue. All these deformations are applied successfully in the FE analysis, as shown schematically in Fig. 4. The flattening is modeled by applying appropriate boundary conditions to the cut strip, namely displacement at the cut edge surface. 
The FE analysis is used to derive the stress-stretch response of the strip that would be measured in a traditional uniaxial testing approach. The stress and the stretch values are both derived as the average values across the strip of $\boldsymbol{\sigma}: \mathbf{N} \otimes \mathbf{N}$ and $\sqrt{\mathbf{C}: \mathbf{N} \otimes \mathbf{N}}$ respectively, where $\mathbf{N}$ is a vector pointing in the direction of the tension axis.

Traditionally, hyperelastic models are formulated with the reference configuration set in the zero load state and without prestretch [25]. An incompressible fourfiber family model in such situation would have a strain energy of the form:

$$
\begin{aligned}
\bar{\psi}= & c\left(\lambda_{r}{ }^{2}+\lambda_{\theta}{ }^{2}+\lambda_{z}{ }^{2}-3\right)+ \\
& \sum_{i=1}^{4} \frac{k_{1}}{2 k_{2}}\left[\exp \left(k_{2}\left(I_{4}^{i}-1\right)^{2}\right)-1\right]
\end{aligned}
$$

where $c, k_{1}>0$ and $k_{2}>0$ are model parameters and $\lambda_{i},(i=r, \theta, z)$, are the three principal stretches. Thus the deformation gradient may be written as $\mathbf{F}=$ $\operatorname{diag}\left[\begin{array}{lll}\lambda_{r} & \lambda_{\theta} & \lambda_{z}\end{array}\right]$.

We propose to fit the stress-strain curves simulated by our FE model with the traditional four-fiber family model written just above. The same orientation angle is used for the diagonal fibers of both models for the sake of simplicity: $\alpha^{3,4}= \pm 45^{\circ}$. Two uniaxial tensile tests are simulated: one with $\mathbf{N}$ aligned in the $\theta$-direction (circumferential) of the artery and the second one with $\mathbf{N}$ aligned in the $z$-direction (axial) of the artery. Then using a least-squares optimization method, parameters $c, k_{1}$ and $k_{2}$ of Eq. 10 are identified. Strips of dimensions $314 \times 160 \times 2 \mathrm{~mm}^{3}$ are modeled, excised from a cylindrical artery with outer diameter $50 \mathrm{~mm}$ having the same material properties as the ones of the human ATAA reported in Tab. 2. The strips are meshed with 22,500 linear hexahedral elements with 26,400 nodes. The deposition stretch of collagen and SMC components is $\sim 1.1$ while the axial deposition stretch of elastin is $\sim 1.3$.

\section{Results}

3.1 Tension-inflation and opening angle response of murine arterial segments

The circumferential component of deposition stretch of elastin is estimated equal to 1.40 and the axial component of deposition stretch of elastin is estimated equal to 1.55 . The $P-D$ and $F-P$ curves obtained with our FE model are shown in Fig. 5. They are compared with analytical counter parts of Bellini et al. [2]. A black spot indicates the reference configuration. A good agreement can be observed between analytical and numerical results. The analytical response was previously validated against experimental data [2]. In Fig. 6 we show the predicted response of an arterial ring to the open angle experiment. The obtained opening angle with the numerical model is $\sim 27.8^{\circ}$, which is just in-between theoretical $\left(\sim 21^{\circ} \pm 3\right)$ and experimental $\left(\sim 29^{\circ} \pm 8\right)$ results of Bellini et al. [2].

\subsection{Patient-specific distribution of stresses in human ATAAs}

From the mesh reconstructed using the preoperative CT scan, the zero pressure geometry of the patientspecific ATAA was derived using our CMT-based FE model. Results are showed in Fig. 7. The distribution of residual stresses in the zero-pressure state shows compression on the luminal side of the artery and tension on the outer side. The stress maps computed at systole are shown in Fig. 8. It is compared with the strain map reported by [52] on the same geometry with a traditional FE model. The traditional model predicts a maximum stress in the wall of $\sim 510 \mathrm{kPa}$ while our new CMT-based FE model predicts it $\sim 600 \mathrm{kPa}$, which is $\sim 17 \%$ higher. Moreover, average nodal displacements of ATAA model using the traditional model and the CMT-based model are $\sim 3.67 \mathrm{~mm}$ and $\sim 1.43 \mathrm{~mm}$, respectively.

Including pulling and twist boundary conditions increases limits of maximum and minimum stresses in the arterial wall. As seen in Fig. 9 CMT-based model predicts a maximum stress of $\sim 650 \mathrm{kPa}$ in the arterial wall.

\subsection{Response of human ATAA strips to uniaxial tensile tests}

Stress-strain curves for both uniaxial tests on human ATAA strips obtained with the CMT-based model are shown in Fig. 10. The flatten strip could be stretched of 1.5 and of 1.28 in the axial (red circles) and circumferential (blue circles) directions, respectively. It can be seen that the curves can be fitted very well with a hyperelastic model using a traditional formulation. The best-fit parameters are $k_{2}=0.1, c=4.65 \mathrm{kPa}$ and $k_{1}=293$ $\mathrm{kPa}$. The values of these parameters are different of the ones used initially in the CMT-based four-fiber family model. The $c$ value of the initial CMT-based model is larger than the one of the traditional model and the $k$ values are smaller. This means that both approaches can predict similar uniaxial tensile responses but with different material parameters. 


\section{Discussion}

\subsection{Main assets of the CMT-based FE model}

We have developed the first FE model based on the constrained mixture theory (CMT) using the Abaqus software and we have shown its potential for patientspecific biomechanical investigations of arteries.

A first advantage of the CMT-based FE model is its ability of predicting the wall stress distribution of arteries in vivo under the effects of physiological pressure by using their CT-scan-based geometry. It is basically accepted that stress analyses of arterial walls using CTscan-based geometries as a reference configuration can lead to errors [13,48], confirmed in recent literature [36, 45]. This is due to the presence of in situ wall stresses existing in the CT-scan-based reference configuration, the artery being loaded by the luminal pressure $[13,35$, $43,48,45]$. Some recent literature [32] proposed an approach for stress analyses which simplifies the biomechanical problem and can use directly CT-scan-based reconstructed geometries, relying on trivial linear elastic finite element computations. Outside of this recent publication, most of the computational approaches for the derivation of wall stresses in arteries relied first on the derivation of the zero-pressure geometry from CTscan-based geometry.

A second advantage of the CMT-based FE model, even if it is not necessary for in situ stress predictions, is its ability for predicting efficiently the zero pressure geometry of an artery from the CT-scan-based geometry. Finding the zero pressure geometry of an artery is a recurrent problem in vascular biomechanics, recently extended to all types of soft tissues by [36]. In [43], a scaling factor was defined on the deformation obtained by applying the diastolic pressure to the CT-based geometry. This permitted to approximate the zero pressure geometry of patient-specific AAAs using a single FE analysis. An improved approach was proposed by [45] for AAAs and extended to ATAAs by [52], with an iterative algorithm to derive the zero pressure geometry, instead of the direct method of [43]. However, there is still noticeable numerical advantages to use our CMT-based FE model over the previously reported methodologies [45] based on incremental upgrading of the deformation gradient. Indeed, the zero pressure geometry is simply the response of the model to a given set of boundary conditions in our model, permitting to derive the solution in a single analysis. It is noteworthy that in the case of patient specific analysis more precaution is necessary because in addition to the patient specific geometry and loading conditions the material properties and prestretch levels may play prominent roles. Therefore, due to large number of material parameters, it is important to ensure that a unique solution exist for the calibration of the circumferential component of the deposition stretch of the elastin.

A third advantage of the CMT-based FE model is its ability for predicting residual stresses, with successful predictions of the opening angle experiment. Residual stresses do exist in the zero-pressure configuration of arteries but they were generally ignored in the previously cited approaches which had also the disadvantage of not being layer specific [45]. Residual stresses were ignored also because the traditional approach for introducing them in a model is to start the analysis from an opened artery, and not to do it reversely as in our model. However, neglecting the residual stresses significantly impacts the distribution of wall stresses at diastole and systole, as shown in Fig. 8 where we compared the two solutions, with and without residual stresses at the zero-pressure state.

A fourth advantage of the CMT-based FE model is to consider the contributions of each constituent of the tissue to its biomechanical response. The constrained mixture theory was herein adopted to include internal stresses which may exist in a reference configuration of a biological tissue $[2,5,50]$. The CMT framework is a mechanobiologically inspired description which can be appropriately employed for any general 3D solid structures under pressure, such as the heart, atria, eye and brain aneurysms among others. It includes the complementary roles of transmural distributions of elastin, collagen fiber families and SMCs. Specifically, our results indicate the primary role of elastin on residual stresses of thick-wall response such as calculated outer diameter and reaction forces of arterial wall versus internal pressure. This is in agreement with experiments by [21] who demonstrated that residual stresses depends primarily on intramural elastin, before collagen or SMC.

A fifth advantage of the CMT-based FE model is to bridge directly the in vivo mechanical behavior of arteries to their behavior observed in vitro after excision. Several authors have tried to identify hyperelastic material parameters in vivo using imaging data and to compare them to the best-fit parameters of stressstrain curves obtained in vitro on the same tissue after excision. However, the reference configuration for the hyperelastic models of those studies was always the zero load geometry of the experimental tests $[47,53]$. We showed here that a different approach can be performed, using the in vivo geometry as a reference configuration for the hyperelastic model and predicting the stress-strain response of in vitro testing after excision using the model. The deposition stretch of each constituent in the reference configuration has a crucial role 
in this response. In a traditional approach with no deposition stretch, collagen fibers are the major contributors to the stress in in vivo conditions. This is rather different with the FE model here, where elastin can have a relatively high contribution to the stress of healthy arteries in the reference configuration in vitro. In a traditional model such as [25], the elastin constituent is represented as a soft matrix in a composite where the collagen fibers are the reinforcements. In the current approach the role of elastin fibers in bearing stresses for in vivo conditions is prominent. Anyway, at the end, both models are able to predict similar stress-strain curves for in vitro uniaxial tensile tests, but with different material parameters. It is yet not trivial to find the parameters of the CMT-based FE model that give an equivalent response to a similar hyperelastic model with no deposition stretch. This may also explain why different maximum stresses are found in Fig. 8 between both approaches.

A sixth advantage of the present model is its mechanobiological foundation. By spliting the contribution of elastin, collagen and SMCs to the wall stress and by taking into account their respective deposition stretches, this permits to: 1- model the transfer of stress from collagen to elastin when elastin gets damaged which is usually the onset of aneurysm growth [59]; 2- vary collagen mass fractions by taking into account the turnover of collagen which can be mechanobiologically driven. In that case the objective is to preserve a constant (homeostatic) stress in the collagen fibers (maximum stress must return to a given target, $\sigma_{0}$, which is homeostatic) [54]; 3- Adapt the deposition stretch of collagen to the configuration in which it is deposited during the growth.

\subsection{Future improvements of the CMT-based FE model}

Despite its promising advantages, the CMT-based FE approach presented in this study still requires research efforts regarding the following points: estimation of the deposition stretches of each constituent from experimental data, regional variations of thickness and material parameters, in vivo assessment of boundary conditions...

Regarding the deposition stretches, Bellini et al. [2] reported bounds for each type of deposition stretches. For collagen fibers the bounds were obtained experimentally using elastase treatment $[3,17]$. Therefore, a value of 1.1 was assigned for collagen fibers and for the passive behavior of SMCs. For elastin, a sensitivity analysis was performed (results not reported) on the effect of the elastic modulus of elastin and of the compressive modulus of collagen and we deduced that they had an impact on the axial and circumferential deposition stretches to be assigned for the elastin in our model. Knowing this, we used values of deposition stretches for elastin below the bounds reported by Bellini et al. [2] but we used a higher value for the elastic modulus of elastin, and a lower compressive modulus of collagen fibers. We found that our combination of material parameters provided a better fit of the experimental data with our numerical model. Moreover, based on existing literature, the compressive modulus of collagen fibers is always assumed negligible compared to the elastic modulus of elastin $[2,12,25,28]$ (see Tables 1 and 2).

Regarding regional variations, in the present work, for patient-specific stress analysis of human ATAAs, the wall thickness is indeed assumed homogeneous. It was measured in vitro on the excised aneurysm of the same patient as explained in $[52,53]$. Therefore, this is an average thickness while it is shown by some authors [9] that thickness varies in an ascending thoracic aorta. Although this can be considered as a limitation of this work, it is possible to include these variations in the present model if data are available. However Choudhury et al. [9] have also shown that these thickness variations are less important in aneurysms than in healthy aortas. The effect of possible variations in the thickness, as well as possible variations in the material properties, would obviously affect the local values of deposition stretches that we have to assign to elastin, as shown in another paper where the regional variations of a CMT-based model were reconstructed on mice aortas [4].

Regarding boundary conditions, consideration of the pulling and twist boundary conditions in patient-specific analysis of ATAAs slightly increases maximum stress compared to the result where only the systolic pressure is applied. A maximum difference of $\sim 50 \mathrm{kPa}$ is calculated here. This shows that the longitudinal stress in the ascending thoracic aorta plays a significant role. However, here we did not assign the patient-specific boundary conditions but some generic values taken from another study [1]. Therefore, it is important for future studies to measure precisely the motion of the aortic root for precise assessment of stresses in ATAAs.

Other points still requiring research efforts for the CMT-based FE approach presented in this study are the following: separation between in-plane contributions of elastin fibers and 3D through-thickness properties to the SEF, implementation of the active role of SMCs, damage... The approach is also tailored for introducing new constituents in the mixture and for changing the mass fractions of existing constituents. Other current developments concern the extension of the Abaqus FE 
model to growth and remodeling predictions in human ATAAs.

\section{Conclusion}

In summary, in this manuscript, a robust computational model based on the constrained mixture theory was presented and its potential was shown for patient-specific biomechanical investigations in aneurysmal aortas of humans. Important developments are currently under progress for the application to patient-specific analysis of aneurysm risk of rupture.

\section{Acknowledgements}

The authors are grateful to the European Research Council for grant ERC-2014-CoG BIOLOCHANICS. The authors would also like to thank Nele Famaey, Chiara Bellini and Jay Humphrey for inspiring discussions related to this work.

\section{Conflict of interest}

There is no conflict of interest.

\section{References}

1. C.J. Beller, M.R. Labrosse, M.J. Thubrikar, and F. Robicsek. Role of aortic root motion in the pathogenesis of aortic dissection. Circulation, 109(6):763-9, 2004.

2. C. Bellini, J. Ferruzzi, S. Roccabianca, E.S. Di Martino, and J.D. Humphrey. A microstructurally motivated model of arterial wall mechanics with mechanobiological implications. Ann. Biomed. Eng., 42(3):488-502, 2014.

3. C. Bellini, N.J. Kristofik, M.R. Bersi, T.R. Kyriakides, and J.D. Humphrey. A hidden structural vulnerability in the thrombospondin-2 deficient aorta increases the propensity to intramural delamination. J Mech Behav Biomed Mater, 71:397-406, 2017.

4. M.R. Bersi, C. Bellini, P. Di Achille, J.D. Humphrey, K. Genovese, and S. Avril. Novel methodology for characterizing regional variations in the material properties of murine aortas. J. Biomech. Eng., 138(7):doi: 10.1115/1.4033674, 2016.

5. L. Cardamone, A. Valentín, J.F. Eberth, and J.D. Humphrey. Origin of axial prestretch and residual stress in arteries. Biomech. Model. Mechanobiol., 8:431-46, 2009.

6. M. Chiquet, L. Gelman, R. Lutz, and S. Maier. From mechanotransduction to extracellular matrix gene expression in fibroblasts. Biochim. Biophys. Acta, 1793:911-20, 2009(5).

7. J.J. Chiu and S. Chien. Effects of disturbed flow on vascular endothelium: pathophysiological basis and clinical perspectives. Physiol. Rev., 91(1):327-87, 2011.
8. J.J. Chiu, S. Usami, and S. Chien. Vascular endothelial responses to altered shear stress: pathologic implications for atherosclerosis. Ann. Med., 41(1):19-28, 2009.

9. N. Choudhury, O. Bouchot, L. Rouleau, D. Tremblay, R. Cartier, J Butany, R Mongrain, and R.L. Leask. Local mechanical and structural properties of healthy and diseased human ascending aorta tissue. Cardiovasc Pathol., 18(2):83-91, 2009.

10. C.J. Chuong and Y.C. Fung. On residual stresses in arteries. J. Biomech., 108:189-192, 1986.

11. E.C. Davis. Elastic lamina growth in the developing mouse aorta. J. Histochem. Cytochem., 43:1115-23, 1995.

12. F.M. Davis, Y. Luo, S. Avril, A. Duprey, and J. Lu. Local mechanical properties of human ascending thoracic aneurysms. J Mech Behav Biomed Mater, 61:235-49, 2016.

13. S. de Putter, B.J.B.M. Wolters, M.C.M. Ruttena, M. Breeuwer, F.A. Gerritsen, and F.N. van de Vosse. Patient-specific initial wall stress in abdominal aortic aneurysms with a backward incremental method. $J$. Biomech., 40:1081-90, 2006.

14. H. Demiray. A layered cylindrical shell model for an aorta. Int. J. Engr. Sci., 29(1):47-54, 1991.

15. K.L. Dorrington and N.G. McCrum. Elastin as rubber. Biopolymers, 16:1201-22, 1977.

16. S. Farzaneh, O. Paseta, and M.J. Gómez-Benito. Multiscale finite element model of growth plate damage during the development of slipped capital femoral epiphysis. Biomech Model Mechanobiol, 14(2):371-85, 2015.

17. J. Ferruzzi, M.J. Collins, A.T. Yeh, and J.D. Humphrey. Mechanical assessment of elastin integrity in fibrillin-1deficient carotid arteries: implications for marfan syndrome. Cardiovasc Re, 92(2):287-9, 2011.

18. YC. Fung. What principle governs the stress distribution in living organism? In: Fung, YC.; Fukada, E.; Wang, JJ., editors. Biomechanics in China, Japan and USA. Science Press; Beijing, pages 1-13, 1983.

19. C.M. García-Herrera and D.J. Celentano. Modelling and numerical simulation of the human aortic arch under in vivo conditions. Biomech Model Mechanobiol., 12(6):1143-54, 2013.

20. T.C. Gasser, M. Auer, F. Labruto, J. Swedenborg, and J. Roy. Biomechanical rupture risk assessment of abdominal aortic aneurysms: model complexity versus predictability of finite element simulations. Eur. J. Vasc. Endovasc., 40:176-185, 2010.

21. S.E. Greenwald, J.E. Moore, A. Rachev, T.P.C. Kane, and J.J. Meister. Experimental investigation of the distribution of residual strains in the artery wall. J. Biomech Engr., 119:438-44, 1997.

22. J.M. Guccione, A.D. McCulloch, and L.K. Waldman. Passive material properties of intact ventricular myocardium determined from a cylindrical model. $J$. Biomech. Eng., 113:42-55, 1991.

23. Hibbit, Karlson, and Sorensen. Abaqus-Theory manual, 6.11-3 edition edition, 2011.

24. G.A. Holzapfel. Nonlinear Solid Mechanics. A Continuum Approach for Engineering. Wiley, Chichester, 2000.

25. G.A. Holzapfel, T.C. Gasser, and R.W. Ogden. A new constitutive framework for arterial wall mechanics and a comparative study of material models. J. Elast., 61:1-48, 2000.

26. G.A. Holzapfel and R.W. Ogden. Constitutive modelling of arteries. Proc. R. Soc. A., 466:1551-97, 2010.

27. L. Horný, M. Netušil, and T. Von̆avková. Axial prestretch and circumferential distensibility in biomechanics of abdominal aorta. Biomech. Model. Mechanobiol., 13(4):783-99, 2014. 
28. J.D. Humphrey. Mechanics of arterial wall: Review and directions. Crit. Rev. Biomed. Eng., 23(1-2):1-162, 1995.

29. J.D. Humphrey. Cardiovascular Solid Mechanics: Cells, Tissues, and Organs. Springer-Verlag NY, 2002.

30. J.D. Humphrey. Vascular adaptation and mechanical homeostasis at tissue, cellular, and sub-cellular levels. Cell Biochem. Biophys., 50(2):53-78, 2008.

31. J.D. Humphrey and K.R. Rajagopal. A constrained mixture model for growth and remodeling of soft tissues. Math. Models Methods Appl. Sci., 12:407-430, 2002.

32. G.R. Joldes, K. Miller, A. Wittek, and B. Doyle. A simple, effective and clinically applicable method to compute abdominal aortic aneurysm wall stress. J. Mech. Behav. Biomed. Mater., 58:139-48, 2016.

33. I. Karsaj, J. Soric, and J.D. Humphrey. A 3-D framework for arterial growth and remodeling in response to altered hemodynamics. Int. J. Eng. Sci., 48(11):1357-72, 2010.

34. B.L. Langille. Arterial remodeling: relation to hemodynamics. Can. J. Physiol. Pharmacol., 74:834-41, 1996.

35. J. Lu, X. Zhou, and M.L. Raghavan. Inverse elastostatic stress analysis in pre-deformed biological structures: Demonstration using abdominal aortic aneurysms. J. Biomech., 40(3):693-6, 2007.

36. S.A. Maas, A. Erdemir, J.P. Halloran, and J.A. Weiss. A general framework for application of prestrain to computational models of biological materials. J. Mech. Behav. Biomed. Mater., 61:499-510, 2016.

37. G. Martufi, A. Satriano, R.D. Moore, D.A. Vorp, and E.S. Di Martino. Local quantification of wall thickness and intraluminal thrombus offer insight into the mechanical properties of the aneurysmal aorta. Ann. Biomed. Eng., 43(8):1759-71, 2015.

38. S.J. Mousavi and M.H. Doweidar. Numerical modeling of cell differentiation and proliferation in force-induced substrates via encapsulated magnetic nanoparticles. Comput Methods Programs Biomed, 130:106-17, 2016.

39. E. Nevo and Y. Lanir. Structural finite deformation model of the left ventricle during diastole and systole. J. Biomech. Eng., 111(4):342-9, 1989.

40. J.H. Omens and Y.C. Fung. Residual strain in rat left ventricle. Circ. Res., 66:37-45, 1990.

41. E. Peña, M.A. Martinez, B. Calvo, and M. Doblaré. On the numerical treatment of initial strains in biological soft tissues. Int. J. Numer. Meth. Engng., 68:836-60, 2006.

42. A. Rachev. Theoretical study of the effect of stressdependent remodeling on arterial geometry under hypertensive conditions. J. Biomech., 30(8):819-27, 1997.

43. M.L. Raghavan, M.A. Baoshun, and M.F. Filinger. Noninvasive determination of zero-pressure geometry of arterial aneurysms. Ann. Biomed. Eng., 34(9):1414-19, 2006.

44. M.K. Rausch and E. Kuhl. On the effect of prestrain and residual stress in thin biological membranes. J. Mech. Phys., 61:1955-69, 2013.

45. F. Riveros, S. Chandra, E.A. Finol, T.C. Gasser, and J.F. Rodriguez. A pull-back algorithm to determine the unloaded vascular geometry in anisotropic hyperelastic aaa passive mechanics. Ann. Biomed. Eng., 41(4):694708, 2013.

46. J.F. Rodriguez, C. Ruiz, M. Doblaré, and G. Holzapfel. Mechanical stresses in abdominal aortic aneurysms: influence of diameter, asymmetry, and material anisotropy. ASME J. Biomech., 130(2):021023, 2008.

47. M. Smoljkić, J. Vander Sloten, P. Segers, and N. Famaey. Non-invasive, energy-based assessment of patient-specific material properties of arterial tissue. Biomech Model Mechanobiol., 14(5):1045-56, 2015.
48. L. Speelman, E.M. Bosboom, G.W. Schurink, J. Buth, M. Breeuwer, M.J. Jacobs, and F. N. van de Vosse. Initial stress and nonlinear material behavior in patient-specific aaa wall stress analysis. J. Biomech., 42(11):1713-19, 2009.

49. J. Stalhand. Determination of human arterial wall parameters from clinical data. Biomech. Model. Mechanobiol., 8(2):141-8, 2009.

50. L.A. Taber and J.D. Humphrey. Stress-modulated growth, residual stress, and vascular heterogeneity. $J$. Biomech. Eng., 123(6):528-35, 2001.

51. J.J. Tomasek, G. Gabbiani, B. Hinz, C. Chaponnier, and R.A. Brown. Myofibroblasts and mechano-regulation of connective tissue remodeling. Nature. Rev., 3:349-63, 2002.

52. O. Trabelsi, F.M. Davis, J.F. Rodriguez-Matas, A. Duprey, and S. Avril. Patient specific stress and rupture analysis of ascending thoracic aneurysms. $J$. Biomech., 48(10):1836-43, 2015.

53. O. Trabelsi, A. Duprey, J.P. Favre, and S. Avril. Predictive models with patient specific material properties for the biomechanical behavior of ascending thoracic aneurysms. Ann. Biomed. Eng., 44(1):84-98, 2016.

54. A. Valentín, J.D. Humphrey, and G.A. Holzapfel. A finite element-based constrained mixture implementation for arterial growth, remodeling, and adaptation: theory and numerical verification. Int. J. Numer. Method. Biomed. Eng., 29(8):822-49, 2013.

55. W.W. von Maltzahn, D. Besdo, and W. Wiemer. Elastic properties of arteries: a nonlinear two-layer cylindrical model. J. Biomech., 14(6):389-97, 1981.

56. W.W. von Maltzahn, R. G. Warriyar, and W. F. Keitzer. Experimental measurements of elastic properties of media and adventitia of bovine carotid arteries. J. Biomech. 17(11):839-47, 1984.

57. H.M. Wang, X.Y. Luo, H. Gao, R.W. Ogden, B.E. Griffith, C. Berry, and T.J. Wang. A modified holzapfelogden law for a residually stressed finite strain model of the human left ventricle in diastole. Biomech. Model. Mechanobiol., 13:99-113, 2014.

58. H. Weisbecker, M.J. Unterberger, and G.A. Holzapfel. Constitutive modelling of arteries considering fibre recruitment and three-dimensional fibre distribution. $J$. $R$. Soc. Interface, 12(105):doi: 10.1098/rsif.2015.0111, 2015.

59. J.S. Wilson and J.D. Humphrey. Evolving anisotropy and degree of elastolytic insult in abdominal aortic aneurysms: Potential clinical relevance? J Biomech, 47(12):2995-3002, 2014.

60. S.L. Woo, J.A. Weiss, M.A. Gomez, and D.A. Hawkins. Measurement of changes in ligament tension with knee motion and skeletal maturation. J. Biomech. Eng., 112:46-51, 1990 
Table 1 Material parameters employed for the murine arterial model. Superscript $t$ and $c$ are tensile and compressive material properties, respectively [2].

\begin{tabular}{ll}
\hline Symbol & Values \\
\hline$\mu^{\mathrm{e}}$ & $337.97[\mathrm{kPa}]$ \\
$D_{1}^{\mathrm{c}_{i}, c}=D_{1}^{\mathrm{m}, c}$ & $18.61[\mathrm{kPa}]$ \\
$D_{2}^{\mathrm{c}_{i}, c}=D_{2}^{\mathrm{m}, c}$ & 1.77 \\
$D_{1}^{\mathrm{c}_{i}, t}$ & $1.38[\mathrm{MPa}]$ \\
$D_{2}^{\mathrm{c}_{i}, t}$ & 6.42 \\
$D_{1}^{\mathrm{m}, t}$ & $32[\mathrm{kPa}]$ \\
$D_{2}^{\mathrm{m}, t}$ & 4.2 \\
$\alpha^{1,2,3,4}$ & $0, \pm \frac{\pi}{4}$ and $\frac{\pi}{2}$ \\
\hline
\end{tabular}

Table 2 Material parameters employed for the human ATAA model. Superscript $t$ and $c$ are tensile and compressive material properties, respectively $[2,5,12]$.

\begin{tabular}{ll}
\hline Symbol & Values \\
\hline$\mu^{\mathrm{e}}$ & $100[\mathrm{kPa}]$ \\
$D_{1}^{\mathrm{c}_{i}, c}=D_{1}^{\mathrm{m}, c}$ & $5[\mathrm{kPa}]$ \\
$D_{2}^{\mathrm{c}_{i}, c}=D_{2}^{\mathrm{m}, c}$ & 0.1 \\
$D_{1}^{\mathrm{c}_{i}, t}$ & $90[\mathrm{KPa}]$ \\
$D_{2}^{\mathrm{c}_{i}, t}$ & 0.85 \\
$D_{1}^{\mathrm{m}, t}$ & $10[\mathrm{kPa}]$ \\
$D_{2}^{\mathrm{m}, t}$ & 0.5 \\
$\alpha^{1,2,3,4}$ & $0, \pm \frac{\pi}{4}$ and $\frac{\pi}{2}$ \\
\hline
\end{tabular}
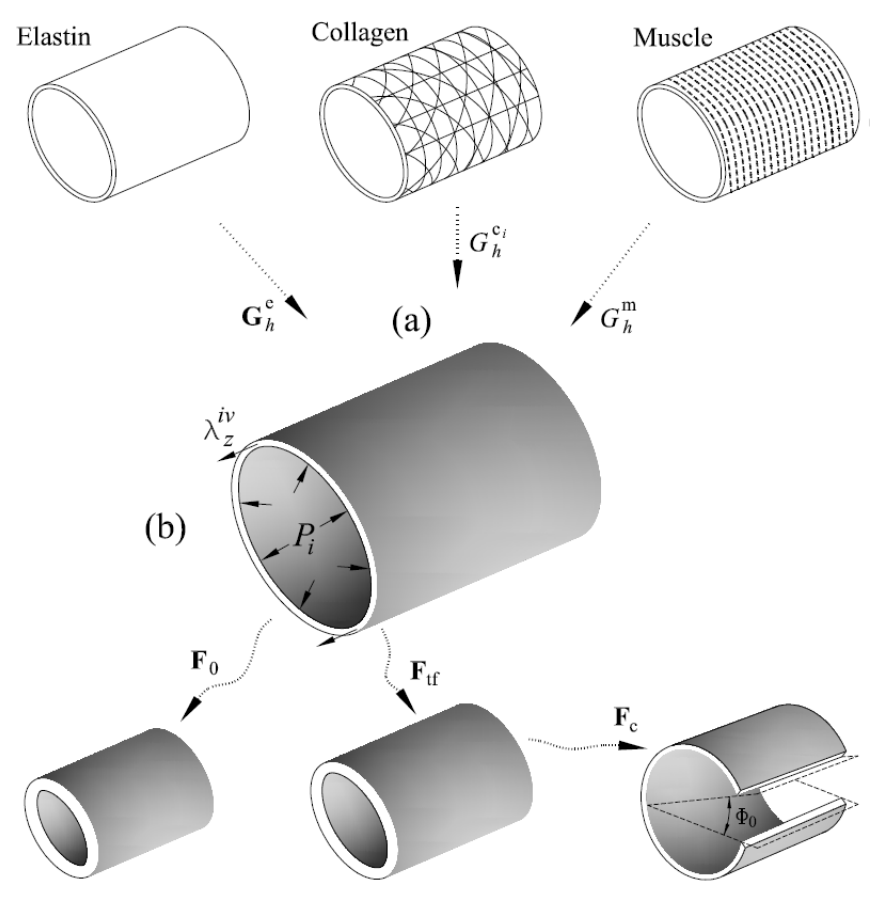

(c)

(d)

(e)

Fig. 1 Schematic illustration of some of the key different configurations relevant for CMT-based models of an arterial segment. a- The arterial wall is composed of elastin, several families of collagen fibers and SMCs. b- Reference configuration under in vivo loading conditions (pressure $P_{i}$ and axial stretch $\lambda_{z}^{\text {iv }}$ ). c- Zero-pressure configuration with no luminal pressure but still with an axial stretch. d- Traction-free configuration which is completely unloaded (the diameter is larger than the one of the zero-pressure configuration). e- Radially-cut configuration with an opening angle of $\Phi_{0} . \mathbf{G}_{h}^{\mathrm{e}}, G_{h}^{\mathrm{c}_{i}}$ and $G_{h}^{\mathrm{m}}$ are deposition and developmental stretches of each load-bearing constituent: elastin, collagen and SMC, respectively, while $\mathbf{F}$ denotes the deformation gradients mapping each configuration from one to another. 


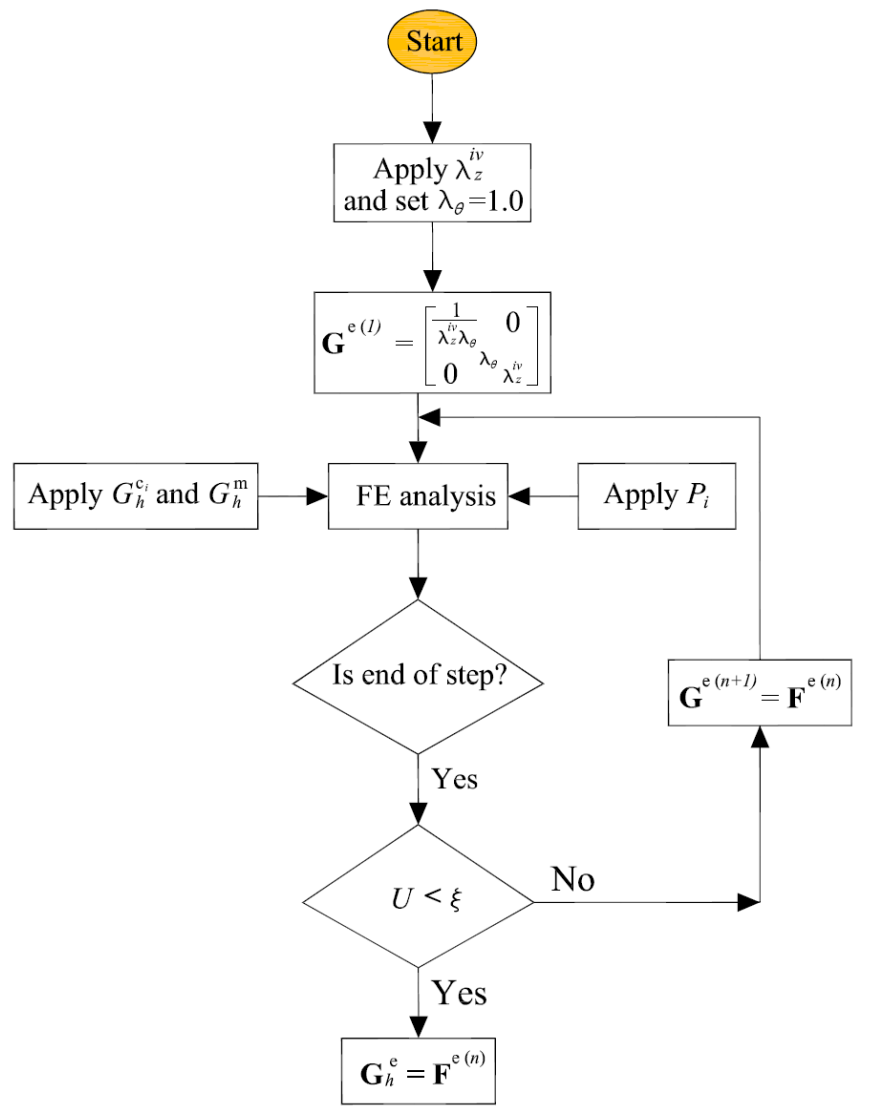

Fig. 2 Flowchart of the algorithm to determine the homeostatic deposition stretch of the elastin component.

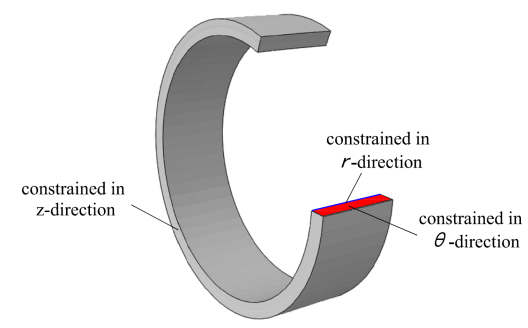

Fig. 3 Assigned boundary conditions for opening angle simulation.

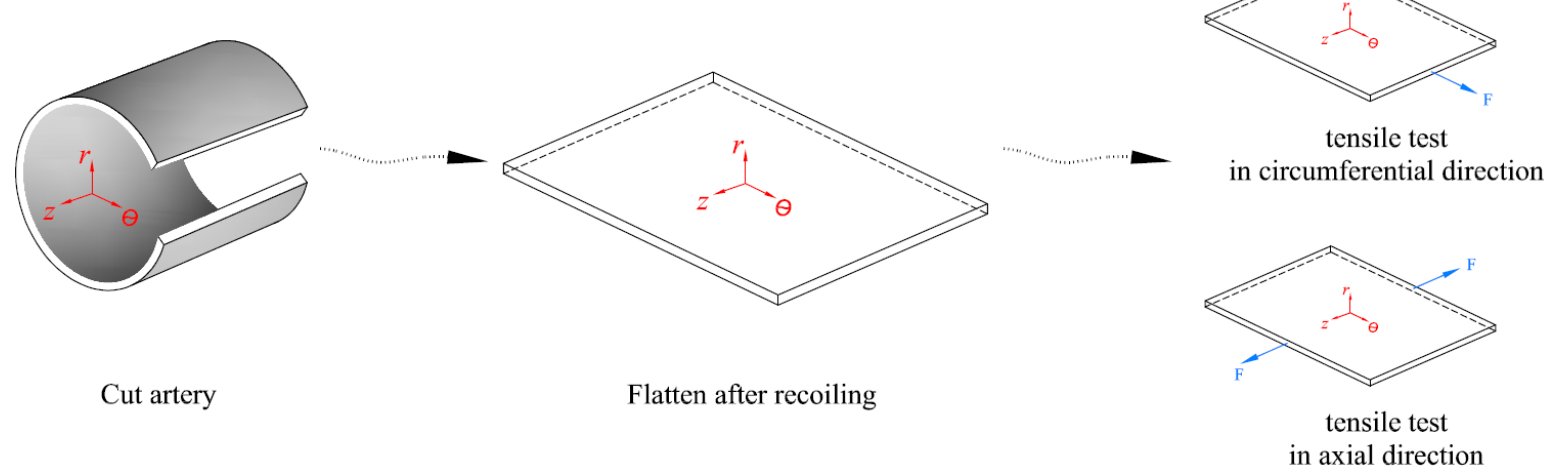

Fig. 4 Schematic description of the process preceding a uniaxial tensile tests, including cutting, flattening and tensile loading. 


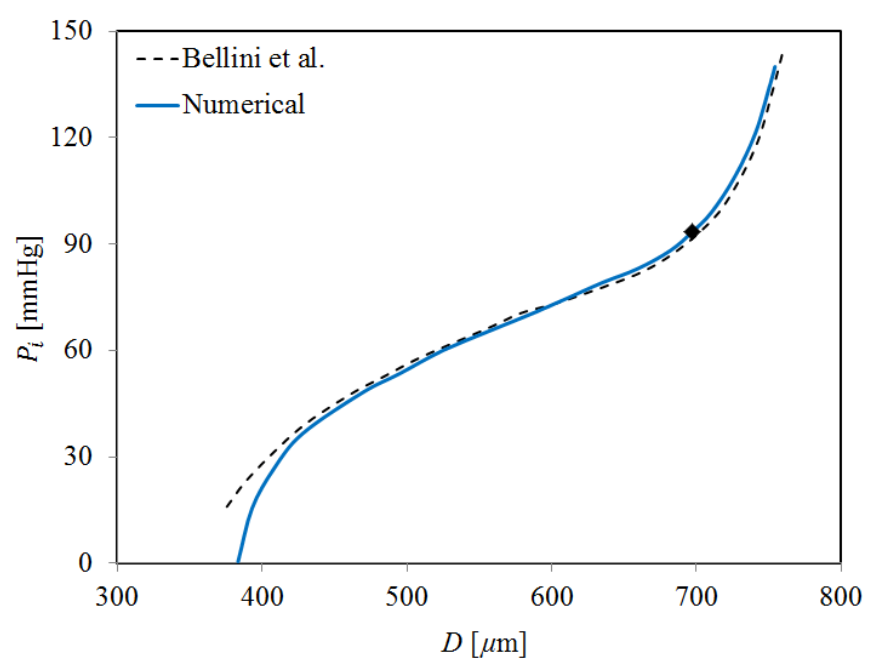

(a)

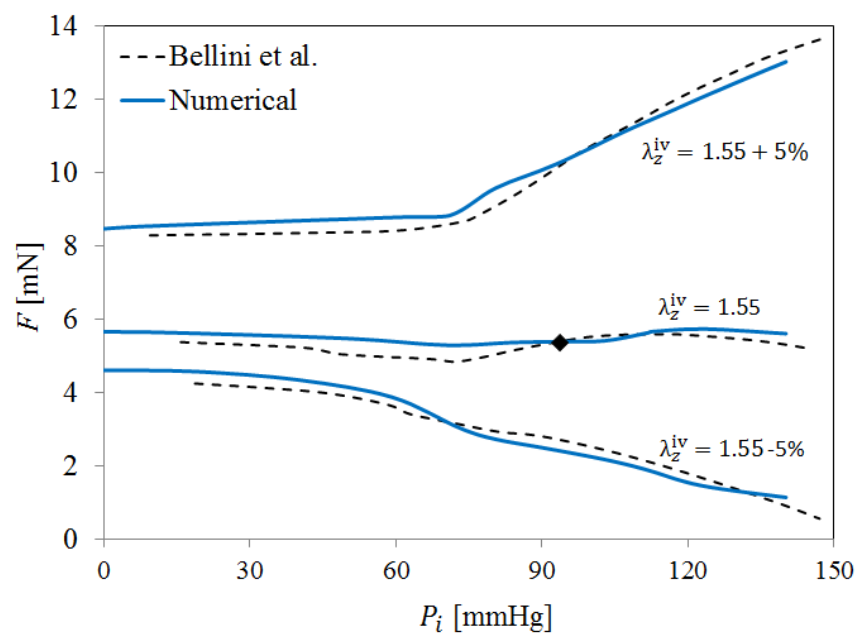

(b)

Fig. $5 P-D\left(\right.$ a) and $F-P\left(\right.$ b) curves at three different axial stretches $\lambda_{z}^{\text {iv }}=1.55 \pm 5 \%$. The $P-D$ curve is shown at $\lambda_{z}^{\text {iv }}=1.55$ only for the sake of facilitating the comparison as the $P-D$ response does not change significantly for other axial stretches [2]. Numerical results are obtained with the present CMT-based FE model whereas analytical results are from [2]. The black spot represents the reference configuration $\left(P_{i}=93 \mathrm{mmHg}\right.$ and $\left.\lambda_{z}^{\mathrm{iv}}=1.55\right)$. 


\section{$\mathrm{S}, \mathrm{S} 22$}

(Avg: 75\%)
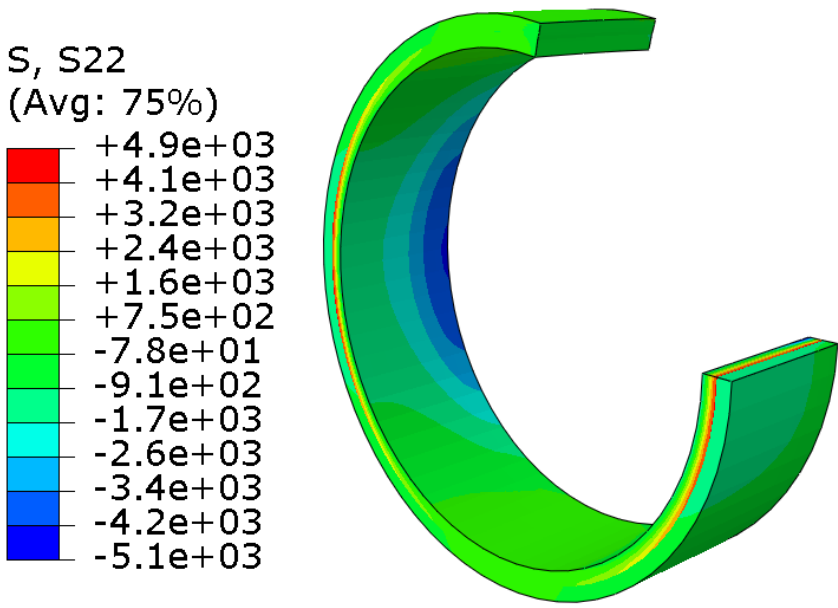

Fig. 6 Response to the opening angle experiment predicted by the numerical model (showing residual circumferential stresses in Pa). The obtained opening angle with the numerical model is $\sim 27.8^{\circ}$ (See also supplemental video), which is just in-between theoretical $\left(\sim 21^{\circ} \pm 3\right)$ and experimental $\left(\sim 29^{\circ} \pm 8\right)$ results of Bellini et al. [2].

\section{$\mathrm{S}, \mathrm{S} 22$}
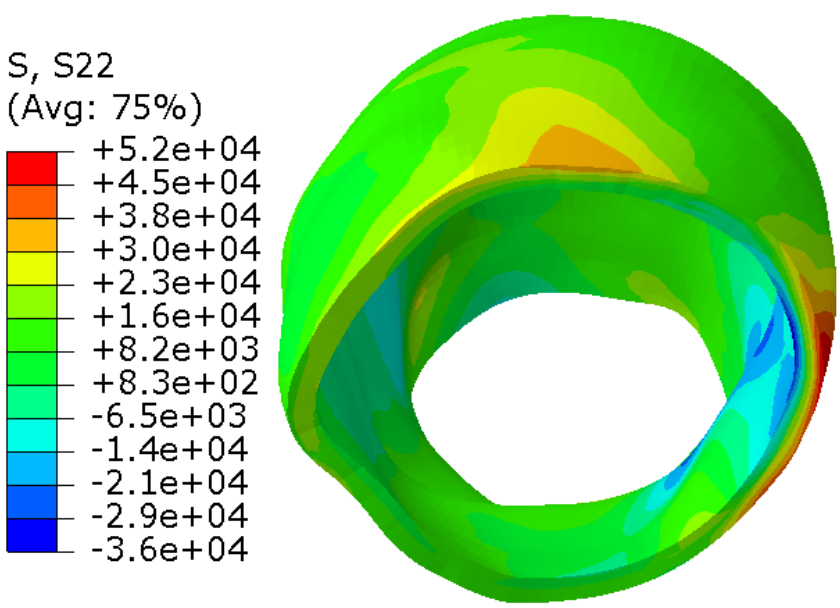

Fig. 7 Zero-pressure configuration including residual circumferential stresses (Pa) for a patient-specific model of human ATAA. 

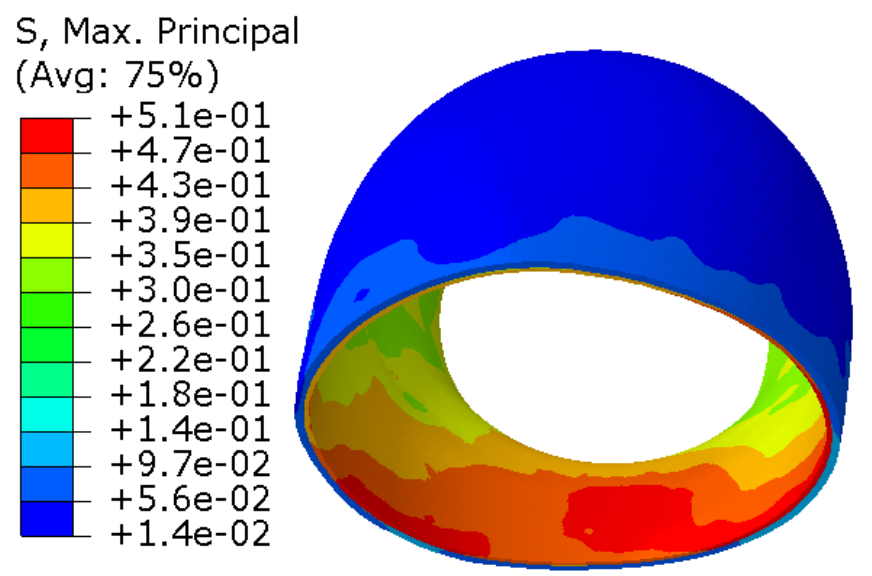

(a)

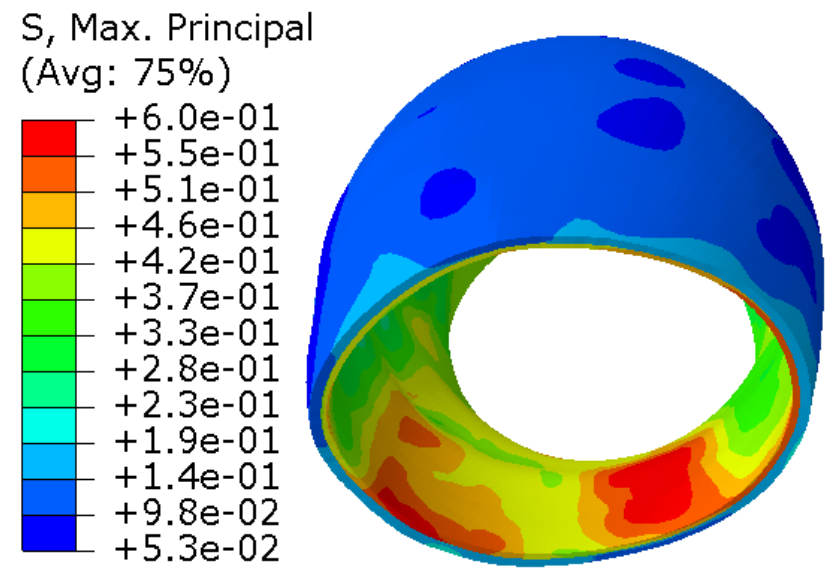

(b)

Fig. 8 Principal stress of the human ATAA model (MPa) for systolic state (16 kPa) using a- traditional methodology and busing CMT-based model.

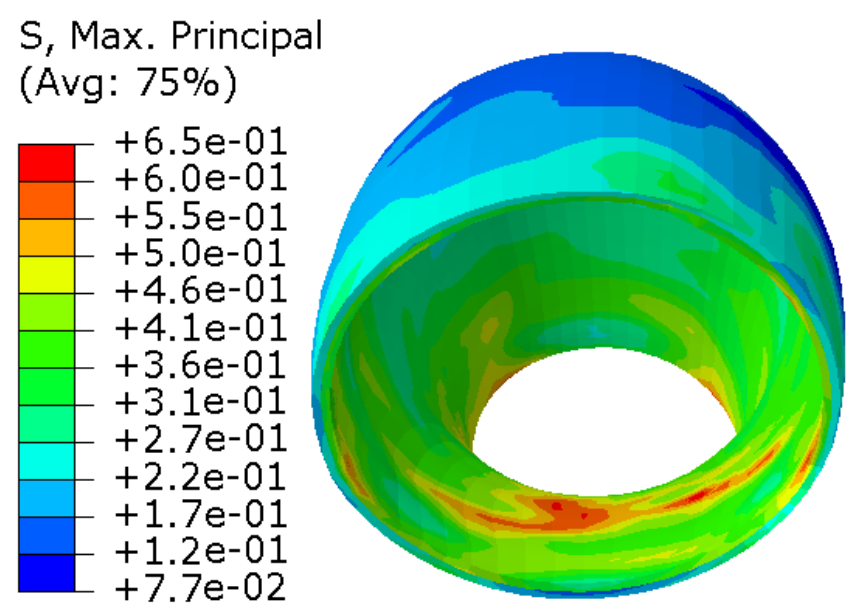

Fig. 9 Principal stress of the human ATAA model (MPa) for systolic state (16 kPa) including pulling and twist boundary conditions. 


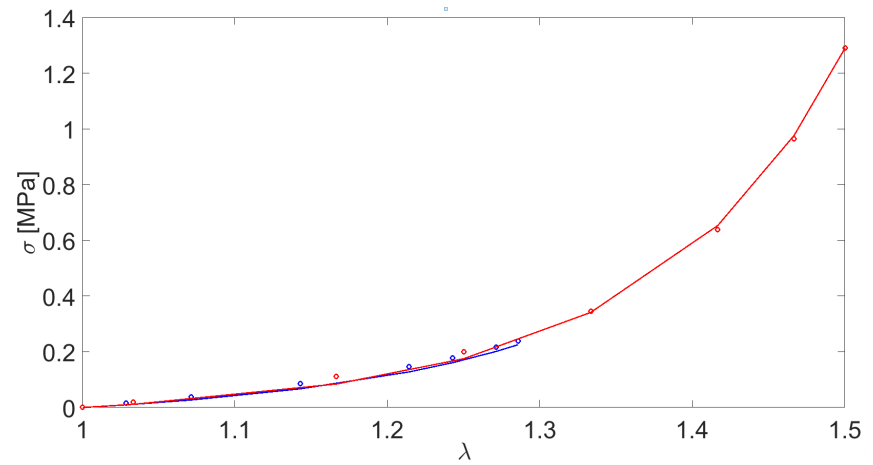

Fig. 10 Stress-strain curves for two uniaxial axial tensile tests on human ATAA strips obtained with the CMT-based model and fitted with a traditional hyperelastic formulation. Red circles and line show the result of the axial direction and the blue ones show the result of the circumferential direction. 